1990-05-01

\title{
Model-based estimation of wind fields over the ocean from wind scatterometer measurements. II. Model parameter estimation
}

David G. Long

david_long@byu.edu

Jerry M. Mendel

Follow this and additional works at: https://scholarsarchive.byu.edu/facpub

Part of the Electrical and Computer Engineering Commons

\section{Original Publication Citation}

Long, D. G., and J. M. Mendel. "Model-Based Estimation of Wind Fields Over the Ocean from Wind Scatterometer Measurements. II. Model Parameter Estimation." Geoscience and Remote Sensing, IEEE Transactions on 28.3 (199): 361-73

\section{BYU ScholarsArchive Citation}

Long, David G. and Mendel, Jerry M., "Model-based estimation of wind fields over the ocean from wind scatterometer measurements. II. Model parameter estimation" (1990). Faculty Publications. 731. https://scholarsarchive.byu.edu/facpub/731 accepted for inclusion in Faculty Publications by an authorized administrator of BYU ScholarsArchive. For more information, please contact ellen_amatangelo@byu.edu. 


\title{
Model-Based Estimation of Wind Fields Over the Ocean From Wind Scatterometer Measurements, Part II: Model Parameter Estimation
}

\author{
DAVID G. LONG, MEMBER, IEEE, AND JERRY M. MENDEL, FELLOW, IEEE
}

\begin{abstract}
A wind scatterometer is a radar remote sensing instrument which measures the wind-dependent radar backscatter of the ocean's surface. From these measurements the near-surface wind over the ocean can be determined. The traditional approach to wind estimation uses only the measurements associated with a given sample point to estimate the wind at that sample point. This point-wise procedure yields nonunique estimates of the wind vector and requires a second "dealiasing" step to select a single estimate. In this second of two papers (see [29]), we demonstrate the feasibility of a new model-based approach to wind field estimation. In the model-based estimation approach we use the parametric model for near-surface mesoscale wind fields developed in Part I [29]. We estimate the parameters of the model from the wind scatterometer measurements; the wind field estimate is then computed from the estimated model parameters. Unlike the traditional point-wise approach, this approach takes advantage of the inherent correlation in the winds at different sample points to more accurately estimate the wind field and resolve directional ambiguity. We compare the accuracy of wind field estimates obtained using the traditional point-wise estimation scheme and our model-based approach using simulated scatterometer measurements from the NASA Scatterometer (NSCAT) $[10]$.
\end{abstract}

\section{INTRODUCTION}

$I^{2}$ N PART I of this series of two papers [29] we introduced a parametric model for near-surface mesoscale wind fields over the ocean. In this paper we use this wind field model as the basis for a fundamentally new estimation-theory-based approach to estimating wind fields over the ocean from wind scatterometer measurements, which we call "'model-based wind field estimation.", We demonstrate the feasibility of this approach and show that it can produce more accurate estimates of the wind field than the traditional approach to wind estimation. It does this by taking advantage of the inherent correlation in the wind over the measurement region. The improved accuracy of the model-based method will expand the utility of winds estimated from wind scatterometer data.

In our model-based estimation approach we formulate an objective function, based on maximum-likelihood (ML) principles, for the model parameters from the scat-

Manuscript received February 16. 1989; revised December 7, 1989

D. G. Long is with the Jet Propulsion Laboratory, California Institute of Technology, MS 300-319, 4800 Oak Grove Drive, Pasadena, CA 91109.

J. M. Mendel is with the Signal and Image Processing Institute, Department of Electrical Engineering-Systems, University of Southern California, Los Angeles, CA 90089.

IEEE Log Number 9034618. terometer measurements. The value of the model parameters which minimizes the objective function is the ML estimate of the model parameters. The estimated wind field is computed from the ML estimate of the model parameters. We refer to our companion paper [29] for the development of the wind field model.

This paper is organized as follows: First, a background in wind scatterometry is provided. This is followed by a discussion of the measurement noise model for the scatterometer. Next, the development of an objective function for the model parameters is presented. Considerations about the optimization of the objective function are then discussed. A simple approach to the optimization of the objective function, based on a gradient search with initial values computed using point-wise wind estimates, is used to demonstrate the feasibility of the model-based wind field estimation approach. Finally, examples are presented which compare the accuracy of the traditional point-wise estimation scheme with our model-based approach; the examples use simulated wind scatterometer measurements from the NASA Scatterometer (NSCAT) [10], [25].

\section{BACKGROUND}

The normalized radar backscatter $\left(\sigma^{\circ}\right)$ (at $\mathrm{Ku}$ band) of the ocean's surface depends on the wind speed and relative azimuth angle between the radar illumination and wind direction in a manner which varies with the incidence angle of the radar on the ocean surface and the radar polarization [3], [27]. The relationship between $\sigma^{\circ}$ and the wind is known as the geophysical model function and will be denoted by $\mathfrak{K}$. A typical example of $\mathfrak{M}$, which we have used in this paper, is the SASS ${ }^{1}$ model function which relates $\sigma^{\circ}$ to the neutral stability wind at $19.5 \mathrm{~m}$ [3]. The model function exhibits a strong $\cos 2 \chi$ dependence of $\sigma^{\circ}$ on wind direction.

The scatterometer measures the $\sigma^{\circ}$ of the ocean's surface. The radar scatterometer transmits a pulse of known power and duration towards the ocean's surface. A portion of this power is reflected, or backscattered, toward the radar. This reflected "signal" power is measured by the scatterometer. The amount of power which is received is a function of the known radar parameters and $\sigma^{\circ}$, which

0196-2892/90/0500-0361\$01.00 (C) 1990 IEEE 
is a function of the wind blowing over the ocean surface [5]. Unfortunately, the power measurement is corrupted by noise. A separate measurement of the noise-only power is made and subtracted from the signal + noise power measurement. This provides the measurement of the backscattered signal power. The $\sigma^{\circ}$ measurement is obtained from the signal power measurement using the wellknown radar equation [27]. $\sigma^{\circ}$ is related to the wind by the geophysical model function $\mathfrak{T}$.

Since $\mathfrak{T}$ has a multivalued inverse, several measurements of $\sigma^{\circ}$ from different azimuth angles must be used to determine the wind vector [27]. The Seasat scatterometer (SASS) obtained $\sigma^{\circ}$ measurements from only two azimuth angles on an irregular sampling grid [7]. Secondgeneration scatterometers such as the NASA Scatterometer (NSCAT) will obtain $\sigma^{\circ}$ measurements from three or more azimuth angles on an equally spaced grid of sample points over the measurement swath. NSCAT will provide measurements of $\sigma^{\circ}$ at a $25-\mathrm{km}$ resolution over a $600-\mathrm{km}$ wide swath on either side of the spacecraft ground track [29, fig. 1], [10], [14]. These noisy measurements of $\sigma^{\circ}$ provide an essentially instantaneous observation of the wind field in the observation swath over the ocean's surface. The problem is to estimate the original wind field at the sample points from the noisy $\sigma^{\circ}$ measurements.

In the traditional approach the noisy $\sigma^{\circ}$ measurements are used in a point-wise estimation scheme in which only the $\sigma^{\circ}$ measurements for a given grid cell are used to estimate the wind for that cell. An objective function (typically based on the likelihood function or least-squares [5]) is formulated using the noisy $\sigma^{\circ}$ measurements and is minimized with respect to the wind speed and direction at the sample point. Unfortunately, due to the nature of $\mathfrak{N}$ the objective function is minimized by several wind vectors. This approach is unable to uniquely estimate the wind vector and several ambiguous wind estimates result for each cell [13]. The multiple estimates are termed ambiguities or aliases [23]. To select a single wind estimate for each cell, a post-estimation procedure, known in the literature as "dealiasing" or "ambiguity removal," is used [23], [28]. Dealiasing procedures have used various ad hoc measures and/or pattern recognition of significant weather features to select a wind vector at each sample point of the wind field and are prone to large systematic errors [1], [23], [25], [28].

Rather than using the traditional point-wise approach to wind estimation, we propose using a model-based estimation procedure to estimate the entire wind field over an entire region of the measurement swath. This new approach can eliminate the need for dealiasing and provides more accurate wind field estimates by taking advantage of the inherent correlation in the wind between different sample points. In this approach we formulate an objective function based on the likelihood function for the model parameters, using the noisy $\sigma^{\circ}$ measurements. The model parameters are estimated by minimizing the objective function. Finally, the estimated wind field is computed from the model parameters. In effect, this procedure per- mits us to estimate the entire sampled wind vector field in one step.

Because model-based wind field estimation uses the wind field model to take advantage of the inherent correlation in the wind across the swath, it is more tolerant of noise in the $\sigma^{\circ}$ measurements than is the pointwise technique. This may permit reductions in the size and weight of future scatterometer instruments by reducing the requirements on the measurement signal-to-noise ratio of the $\sigma^{\circ}$ measurements, permitting smaller transmitters, antennas, etc.

The pointwise wind estimation approach requires that there be co-located measurements of $\sigma^{\circ}$ from at least two different azimuth angles in order to retrieve the wind [25]. Where there are missing $\sigma^{\circ}$ measurements (due, for example, to calibration cycles), the wind cannot be retrieved. This produces "holes" in the estimated wind fields and complicated dealiasing. In the model-based approach a wind vector is determined at every point of the swath, even when there are missing $\sigma^{\circ}$ measurements; hence, there are fewer data gaps in the retrieved winds. Furthermore, all of the available $\sigma^{\circ}$ measurements can be used at all sample points even if there is only one $\sigma^{\circ}$ measurement in a given wind vector cell (for which a pointwise wind estimate cannot be determined). These additional measurements help reduce the overall wind field estimate error.

\section{The Scatterometer Measurement Process}

The wind scatterometer provides essentially instantaneous measurements of $\sigma^{\circ}$ over a grid of sample points in each of the measurement swaths (refer to [29, fig. 1]). For NSCAT, the sample points or resolution "cells" are separated by $25 \mathrm{~km}$. At each sample point noisy measurements, denoted $z(k)$, of the true $\sigma^{\circ}$, denoted $\sigma^{\circ}(k)$, are obtained for each of the antenna beam azimuth angle/polarizations. In the case of NSCAT, the center antenna beam is dual-polarized so that there are three azimuth angles but four observations of $\sigma^{\circ}[14]$; hence, $k$ ranges from 1 to 4 . Occasionally, due to calibration cycles and co-registration errors, there may be missing $\sigma^{\circ}$ measurements for some sample points.

The NSCAT instrument is designed to provide $\sigma^{\circ}$ measurements at $25-\mathrm{km}$ resolution. However, the wind is typically estimated at a $50-\mathrm{km}$ resolution by first resampling the $25-\mathrm{km}$ resolution $\sigma^{\circ}$ measurements onto a $50-\mathrm{km}$ grid. For each grid point, the wind is estimated using pointwise wind estimation followed by dealiasing [10]. Our method can be applied to provide wind estimation at both $25-$ and $50-\mathrm{km}$ resolution [15], though we present only $50-\mathrm{km}$ resolution results in this paper.

$\sigma^{\circ}(k)$ is related to the wind at the sample point by the geophysical model function,

$$
\sigma^{\circ}(k)=\mathscr{N}\{(u, v), k\}
$$

where $u$ and $v$ are the components of the wind at the sample point, and the $k$ argument in $\mathfrak{T}$ subsumes the depen- 
dence of $\sigma^{\circ}$ on the antenna observation azimuth angle $\Psi$, the incidence angle $\theta$, and polarization $p$. Note that the relative azimuth angle $\chi$ is

$$
\chi=\Psi-\phi
$$

where $\phi$ is the wind direction.

Let us consider the measurement process for a particular observation $k$ at a given sample point. The noise model for the noisy measurement $z(k)$ of the true $\sigma^{\circ}$ value may be expressed as

$$
z(k)=\sigma^{\circ}(k)+\nu(k)
$$

where $\nu(k)$ is a zero mean Gaussian random variable whose variance is dependent on the true $\sigma^{\circ}, \sigma^{\circ}(k)$, the measurement geometry, the scatterometer design, and includes the statistical fluctuations in the return due to scatter from the ocean surface [13], [27]. The variance of $z(k)$ is

$$
\operatorname{Var}[z(k)]=\alpha^{2}(k) \sigma^{\circ 2}(k)+\beta^{2}(k) \sigma^{\circ}(k)+\gamma^{2}(k)
$$

where $\alpha(k), \beta(k)$, and $\gamma(k)$ depend on the measurement geometry and scatterometer design [5], [13]. Additional discussions of the scatterometer measurement model are found in [6], [4], [13], [15], [27].

The conditional probability density of $z(k)$, given $\sigma^{\circ}(k)$, is

$$
\begin{aligned}
p\left(z(k) \mid \sigma^{\circ}(k)\right) & \\
= & \frac{1}{\sqrt{2 \pi}} \frac{1}{\sqrt{\operatorname{Var}[z(k)]}} \\
& \cdot \exp \left\{-\frac{1}{2}\left[z(k)-\sigma^{\circ}(k)\right]^{2} / \operatorname{Var}[z(k)]\right\} .
\end{aligned}
$$

At a given sample point the log-likelihood function (ignoring constants) $L(u, v)$ can be expressed as [5], [13], [25]

$$
\begin{aligned}
L(u, v)= & -\sum_{k=1}^{L_{p}}\{\log \{\operatorname{Var}[z(k)]\} \\
& \left.+\left[z(k)-s^{2}(k)\right]^{2} / \operatorname{Var}[z(k)]\right\}
\end{aligned}
$$

where $L_{p}$ is the number of measurements at the sample point.

\section{Model-Based Wind Field Estimation}

The role of the wind field model in model-based wind field estimation is to provide a description of the wind field over the scatterometer measurement swath at a fixed instant of time and resolution of from 25 to $50 \mathrm{~km}$ (corresponding to the scatterometer sampling resolution). In Part I [29] we developed a model for the wind field $\bar{W}$ over a square region $\mathscr{L}$ with $N$ samples on a side having the general form:

$$
\bar{W}=F \bar{X}
$$

where $\bar{W}$ contains the components of the sampled wind field over the region in lexicographic order (see Part I [29]), $F$ is a $2 N^{2} \times N_{u}$ dimension constant matrix of full rank, and $\bar{X}$ is an $N_{u}$-dimensional model parameter vector. The value of $N_{u}$ depends on the order of the wind field model. Typically, we choose $N=8$ or $N=12$ for the PBC model (see Part I [29]), with $M_{l}=8$ and $M_{c}=M_{d}$ $=2, N_{u}=20$.

This model readily lends itself to the following parameter estimation formulation: The model parameters in $\bar{X}$ are estimated directly from the noisy $\sigma^{\circ}$ measurements, and then the wind field is computed from the estimated model parameters.

Given an arbitrary wind field, denoted by $\bar{W}_{t}$, the leastsquares fit $\bar{X}$ of the model parameters to $\bar{W}_{t}$ is

$$
\bar{X}=F^{\dagger} \bar{W}_{t}
$$

where $F^{\dagger}=\left(F^{T} F\right)^{-1} F^{T}$ is the pseudo-inverse of $F$. The wind field computed from the model parameter vector can then be computed using (7). Note that the components $u_{i, j}$ and $v_{i, j}$ of the wind vector at the sample point $(i, j)$ within $\mathscr{L}$ are

$$
\begin{aligned}
& u_{i, j}=\bar{W}_{n}=(F \bar{X})_{n} \\
& v_{i, j}=\bar{W}_{N^{2}+n}=(F \bar{X})_{N^{2}+n}
\end{aligned}
$$

where $n=(j-1)+i$ is the lexicographic index corresponding to $i, j$, where $i$ and $j$ range from 1 to $N$.

\section{Objective Function Formulation}

Following a standard approach in estimation theory, we define an objective function for the model parameters from the available measurements [19], [20]. The parameter estimate is determined by minimizing the objective function. In our approach, we base the objective function on the maximum-likelihood principle [20].

The true value of $\sigma^{\circ}$, denoted $\sigma_{n}^{\circ}(k)$ (corresponding to the $k$ th observation of the $n$th sample point identified by the lexicographic index $n$ ), can be expressed as

$$
\begin{aligned}
\sigma_{n}^{\circ}(k) & =\mathfrak{T}\left\{\left(u_{n}, v_{n}\right), k\right\} \\
& =\mathfrak{T}\left\{\left((F \bar{X})_{n},(F \bar{X})_{N^{2}+n}\right), k\right\} .
\end{aligned}
$$

The conditional probability density of the $k$ th measurement of $\sigma^{\circ}$ at $n, z_{n}(k)$, given $\bar{X}$, is (refer to (5))

$$
\begin{aligned}
p\left(z_{n}(k) \mid \bar{X}\right) & \\
= & \frac{1}{\sqrt{2 \pi}} \frac{1}{\sqrt{\operatorname{Var}\left[z_{n}(k)\right]}} \\
& \cdot \exp \left\{-\frac{1}{2}\left[z_{n}(k)-\sigma_{n}^{\circ}(k)\right]^{2} / \operatorname{Var}\left[z_{n}(k)\right]\right\}
\end{aligned}
$$

where $\sigma_{n}^{\circ}(k)$ is given by (11) and $\operatorname{Var}\left[z_{n}(k)\right]$ is given by (4). There are $L_{n}$ measurements available at the sample 
point $n ; L_{n}$ may vary from its nominal value (of 4 for NSCAT) due to missing measurements.

The measurement noise is assumed to be independent for each $z_{n}(k)$. It follows that the log likelihood function $l(\bar{X})$ for $\bar{X}$, given all the measurements $z_{n}(k)$, is

$$
l(\bar{X})=\sum_{n=1}^{N^{2}} \sum_{k=1}^{L_{n}} \log p\left(z_{n}(k) \mid \bar{X}\right) .
$$

Disregarding any constants, $l(\bar{X})$ can be written as

$$
\begin{aligned}
l(\bar{X})= & -\sum_{n=1}^{N^{2}} \sum_{k=1}^{L_{n}}\left\{\log \left\{\operatorname{Var}\left[z_{n}(k)\right]\right\}\right. \\
& \left.+\left[z_{n}(k)-\sigma_{n}^{\circ}(k)\right]^{2} / \operatorname{Var}\left[z_{n}(k)\right]\right\}
\end{aligned}
$$

where $\sigma_{n}^{\circ}(k)$ is given by $(11)$ and $\operatorname{Var}\left[z_{n}(k)\right]$ is given by (4). We define the objective function $J(\bar{X})$ as the negative of the log likelihood function. The maximum-likelihood estimate of $\bar{X}$ is obtained by minimizing $J(\bar{X})$.

The estimation theory concept of "identifiability" indicates whether or not an estimated quantity can be uniquely determined from the available measurements [13], [20]. It can be shown that $\bar{X}$ is set-wise identifiable from the measurements of $\sigma^{\circ}[15]$. An outline of the proof is given here.

For an arbitrary $\bar{W}$ there exists a unique corresponding $\bar{X}=F^{\dagger} \bar{W}$, and, for a given $\bar{X}$, there is a corresponding $\bar{W}$ $=F \bar{X}$; consequently, showing that $\bar{X}$ is identifiable from the noisy $\sigma^{\circ}$ measurements is essentially equivalent to showing that $\bar{W}$ is identifiable from the noisy $\sigma^{\circ}$ measurements.

Due to the nature of the model function (i.e., the upwind $\sigma^{\circ}$ has the same value as the downwind $\sigma^{\circ}$ ), there may be several possible wind fields (with corresponding model parameter vectors) that could have given rise to the observed $\sigma^{\circ}$ measurements. Let the set $D_{f}^{c}$ denote the set of all $\bar{X}$ which give rise to the same true $\sigma^{\circ}$ observations; i.e.,

$$
D_{f}^{c} \triangleq\left\{\bar{X} \mid \mathfrak{N}\left\{(F \bar{X})_{n}, k\right\}=\mathfrak{N}\left\{\left(F \bar{X}_{t}\right)_{n}, k\right\} \forall n, k\right\}
$$

where $\bar{X}_{t}$ denotes the true value of $\bar{X}$. The set $D_{f}^{c}$ can have one or more members. In general, however, $D_{f}^{c}$ contains only a single member. In order for $D_{f}^{c}$ to contain multiple members each of the wind fields corresponding to the different $\bar{X}$ must have wind vectors at all corresponding sample points that produce exactly the same values of $\sigma_{n}^{\circ}(k)$ for all $k$ and $n$. For a given wind field, this is a highly unlikely occurrence.

To show identifiability of the model parameters in model-based wind field estimation, we need to show that as $T \rightarrow \infty$ (corresponding to a longer and longer measurement), the locations of global minima of $J(u, v)$ converge in probability to the members of $D_{f}^{c}$. We apply the technique used in the point-wise case [13] to show (i) that $J(u, v) / T$ converges in the mean square to $E[J(u$, $v) / T]$, and (ii) the maximum-likelihood estimates of $\bar{X}$ converge in probability to some element $\bar{X}_{1} \in D_{f}^{c}$; i.e.,

$$
\lim _{T \rightarrow \infty} E\left[\frac{J\left(\bar{X}_{1}\right)}{T}\right]=\min _{\bar{X}} \lim _{T \rightarrow \infty} E\left[\frac{J(\bar{X})}{T}\right] .
$$

Details of the proof are given in [15].

\section{Vi. Objective Function Optimization}

Since $\mathfrak{K}$ is a tabular function, a closed form for the minimum of $J(\bar{X})$ is not available; hence $J(\bar{X})$ must be numerically optimized. Unfortunately, the objective function can be difficult to optimize due to the nonlinear properties it inherits from the nature of $\mathfrak{N}$ and the dimensionality of the problem. The objective function has numerous local minima with the possibility of several global minima. When multiple minima occur, the maximumlikelihood estimate is a set. Selection of a single solution cannot be obtained from just the $\sigma^{\circ}$ measurements. In this event an additional step is required to select a single solution. This situation is discussed below.

Classic nonlinear minimization algorithms include stochastic algorithms [18], [21] such as simulated annealing [8] and various gradient search techniques [2], [16]. Recently, Slump and Hoenders [26] developed a technique which is capable of locating all the global minima of an objective function; however, the computation requirements of their method for a large dimensional problem such as ours prohibits its use. While random optimization techniques are able to locate global minima, they may require an excessive number of function evaluations to find even a single global minimum [18]. Multiple global minima are determined by restarting several times. Gradientbased optimization algorithms can get stuck in a local minimum and fail to find a global minimum. Starting the search at different initial values can be used to find multiple minima with similar objective function values, although there is often no way to determine if the minima are global.

In spite of its limitations, a gradient search algorithm can be used successfully if appropriate initial values can be determined. Such initial values can be computed for the optimization of the field-wise objective function using the results of the traditional point-wise approach to wind field estimation. We will describe one such approach to the initial value computation in the sequel. This optimization technique is relatively simplistic; better results can be obtained with more sophisticated initial-value and optimization algorithms [15]. Even so, our model-based wind field estimates that were obtained using this simplistic optimization approach are more accurate than the traditional point-wise wind field estimates. Our simple optimization approach is adopted merely for the purpose of demonstrating the feasibility of model-based estimation.

\section{Field-Wise Dealiasing}

Due to the nature of the geophysical model function $\mathfrak{M}$, it is possible to have several global minima of the objec- 
tive function (see the discussion above on identifiability). When this occurs the maximum-likelihood estimate is a set, and a single estimate cannot be determined from the $\sigma^{\circ}$ measurements alone. In this event, an additional step is required to select a single solution. Such a procedure might be termed "field-wise dealiasing" because of its seeming similarity to the "point-wise dealiasing" which is always required by the point-wise wind estimation approach. There is, however, a distinct difference between field-wise and point-wise dealiasing: In the point-wise case, the problem is to choose from two to six possible solutions at each sample point (of which there are $N^{2}$ in an $N \times N$ region), while in the field-wise case we need only choose between a few fields. The use of auxiliary data, climatological data, and/or continuity considerations with adjacent regions can easily resolve the fieldwise ambiguity problem.

Since the occurrence of multiple global minima is very rare and our goal is only to show the feasibility of the model-based wind estimation approach, we will not here consider field-wise dealiasing any further here.

\section{ViII. Initial Value Computation and Optimization}

In order to use a gradient-search optimization algorithm successfully in this application, proper selection of the initial values is crucial. In this section we describe a technique for computing an initial value wind field based on a point-wise wind field estimate, followed by a medianfilter-based dealiasing algorithm. The initial value model parameter vector is computed using (8). Alternate initial value computation schemes are described in [15]. While dealiasing errors can result in very poor quality initial values, this approach is suitable for demonstrating the feasibility of model-based wind field estimation. This approach has the additional advantage of providing both point-wise and model-based wind field estimates for comparison purposes.

\section{A. Point-Wise Wind Estimation}

In the traditional approach to wind estimation, only the measurements of $\sigma^{\circ}$ associated with each sample point of the measurement swath are used to estimate the wind vector at the sample point; i.e., the wind is estimated on a point-wise basis. Unfortunately, on a point-wise basis the wind vector is only set-wise identifiable from the $\sigma^{\circ}$ measurements; i.e., there is not a unique wind estimate (see [13]). Due to the nature of the $\mathfrak{K}$, there are several local minima (two to six) in the point-wise maximum-likelihood objective function (6) that are near-global. Collectively, the wind vectors corresponding to these local minima are known as the noisy ambiguity set. Typically, the aliases have similar wind speed but differ significantly in direction. While all of the aliases have similar values of the objective function, they may be "ranked" according to the objective function value; hence the "first" alias (corresponding to the alias with the lowest objective function value) would be the classic ML estimate of the wind. However, since other members of the ambiguity set are very often closer to the true wind than is the first alias, the traditional approach has been to retain all of the aliases and use dealiasing or ambiguity removal to select a unique wind estimate. Traditionally, dealiasing has been based on various ad hoc considerations, including pattern recognition of significant wind field features, continuity considerations, etc. [23], [28].

As a graphic example of point-wise and model-based wind estimation, consider Fig. 1(a), which shows a section of a wind field, taken from [29, fig. 5], sampled at $25 \mathrm{~km}$ over a $300 \times 300$ region. This is the true wind field. A least-squares fit of the model parameters to this true field can be made using (8). The resulting model parameter vector is known as the "true model parameters." The wind field computed from these true model parameters, using (7), is termed the "true model field" " and appears to be virtually identical to the true field shown in Fig. 1(a). The model used is the PBC model (cf. [29, figs. 3 and 4]) with $N=12, M_{c}=M_{d}=2$, and $M_{l}=8\left(N_{u}\right.$ $=20$ ). Better fits can be achieved with a higher-order model at the expense of additional unknowns $N_{u}$. Simulated $\sigma^{\circ}$ measurements from this field were generated based on the NSCAT scatterometer design [15], [25]; the resulting point-wise ambiguity sets are plotted in Fig. 1(b).

\section{B. Dealiasing}

We have used the baseline NSCAT dealiasing algorithm which is based on two-dimensional median filtering [25]. Unlike a low-pass filter, the median filter does not smooth edges or boundaries in the data. Performance of the dealiasing algorithm is dependent on the underlying wind field, any missing measurements, the $\sigma^{\circ}$ measurements, and the median filter window size. Fig. 1(c) illustrates the dealiased wind field corresponding to Fig. 1(b). Careful examination will reveal that in this example the dealiasing algorithm was unable to correctly select the ambiguity closest to the true wind vector at every sample point (see also the example in Figs. 4-9 discussed below). Compare the dealiased wind field in Fig. 1(c) to the true wind field in Fig. 1(a); the dealiased wind field appears to be a "noisy" estimate of Fig. 1(a).

While overall performance of the median filter-based dealiasing is good in moderate-to-high wind speeds, ambiguity selection errors tend to be highly clustered, particularly in low wind-speed regions that contain significantly more dealiasing errors [25]. The selection error dependence on wind speed is typical of dealiasing algorithms [23], [28].

\section{Model Parameter Initial Value Computation}

The dealiasing algorithm is applied over as large a region as possible to minimize edge effects. The dealiased field is segmented into adjacent $N \times N$ (typically, $N=$ 12 ) regions, and the least-squares fit of the model parameters for each region is computed using (8). Missing dealiased wind estimates at a given sample point are filled with an average of adjacent dealiased winds. For each re- 


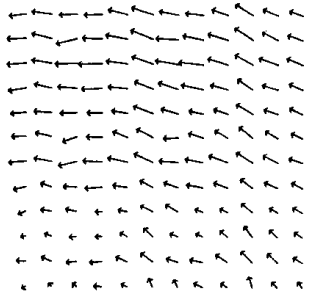

(a)

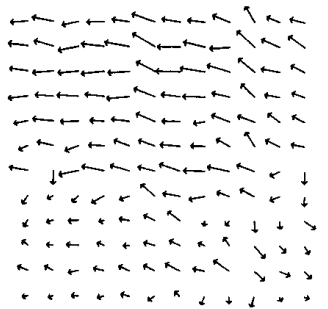

(c)

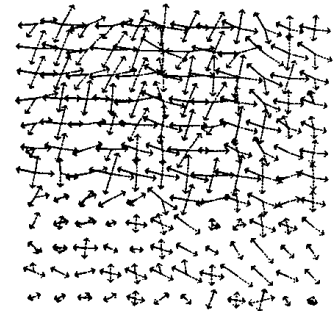

(b)

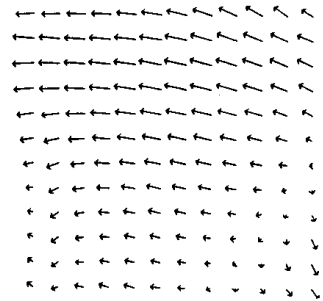

(d)

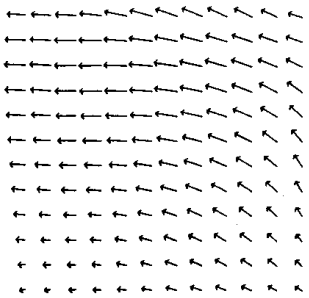

(e)
Fig. 1. Application of model-based wind field estimation for a given 300 $\times 300 \mathrm{~km}$ region with $h=25 \mathrm{~km}$. A vector length equal to the sample spacing corresponds to $15 \mathrm{~m} / \mathrm{s}$. (a) An example of wind field. (b) Noisy ambiguity sets resulting from point-wise estimation of (a) using simulated $\sigma^{\circ}$ measurements from NSCAT. Solid lines indicate the ambiguity closest to the true wind vector. (c) The result of median-filter-based dealiasing to (b). (d) The initial value wind field computed from (c) using the computational method described in the text. (e) The wind field computed using the optimized model parameters. This is the model-based wind field estimate.

gion, the model parameters computed using this leastsquares fit to the dealiased wind field are referred to as "initial value model parameters." These are used as the initial values to begin the optimization of the objective function with respect to the model parameters for the particular region. The wind field computed from the initial value parameters using (7) is known as the "initial wind field." The model-based estimate of the wind field is computed from the optimized model parameters.

Fig. 1(d) illustrates the initial wind field computed from the least-squares fit of the model parameters to the dealiased wind field in Fig. 1(c). Compare this initial value wind field in Fig. 1(d) to the true wind field in Fig. 1(a) and the dealiased wind field in Fig. 1(c). We note that Fig. 1(d) makes a better estimate of the true wind field than Fig. 1(c) even without further optimization. This observation can be used to reduce the computation required to obtain the model-based wind field estimate (discussed
TABLE I

Wind Field Estimate ERror

\begin{tabular}{|c|c|c|c|c|}
\hline Field & $\begin{array}{c}\text { Figure } \\
\text { Number }\end{array}$ & Vector $(\mathrm{m} / \mathrm{s})$ & $\begin{array}{c}\text { RMS Error } \\
\text { Direction (deg) }\end{array}$ & Speed $(\mathrm{m} / \mathrm{s})$ \\
\hline True Model & - & 0.99 & 11.62 & 0.65 \\
\hline Point-wise Closest & $\mathrm{lb}$ & 1.33 & 21.63 & 0.58 \\
\hline Point-wise Dealiased & Ic & 2.42 & 54.51 & 0.62 \\
\hline Initial Value & Id & 2.04 & 47.25 & 1.13 \\
\hline Model-based Estimate & le & 1.16 & 13.81 & 0.74 \\
\hline
\end{tabular}

below). Table I provides a summary of the root-meansquare (rms) difference in wind speed, direction, and the magnitude of the vector difference between the true field and each case in Fig. 1.

The difference (known as the model-fit error) between the initial value wind field and dealiased wind field (from which the initial value wind was computed via a leastsquares fit of the model parameters) provides a measure of the accuracy of the dealiasing. When the dealiasing algorithm chooses the correct ambiguity at each sample point, the initial value rms model-fit direction error is generally small. However, when there are clustered dealiasing errors, the initial value rms model-fit direction error is generally much larger. This leads to a simple threshold-based dealiasing algorithm accuracy check. When the rms initial value model-fit direction error is above a threshold (typically $15^{\circ}-20^{\circ}$, depending upon wind speed), the accuracy of the dealiasing for the region may be considered suspect. The model-fit errors for the initial value field in Fig. 1(d) relative to the dealiased field in Fig. 1(d) are $1.76 \mathrm{~m} / \mathrm{s}$ rms vector error, $36^{\circ} \mathrm{rms} \mathrm{di-}$ rection error, and $1.15 \mathrm{~m} / \mathrm{s}$ rms speed error.

\section{Gradient-Search Optimization}

Given initial values, a gradient-search algorithm has been used to perform the optimization of $J(\bar{X})$. For this purpose we have used the standard IMSL routine IMING for nonlinear optimization, which uses quasi-Newton gradient optimization. Starting with the initial value field in Fig. 1(d), the wind field computed from the optimized model parameters, known as the "optimized wind field," is shown in Fig. 1(e). Compare Fig. 1(f) with the dealiased point-wise wind field estimate in Fig. 1(c). From these figures and Table I we see that the model-based estimate is superior to the point-wise estimate; it is less "noisy" and has a smaller rms vector and direction error. The model-based estimate has a slightly larger rms speed error, since the model-based approach effectively minimizes the rms vector error at the possible expense of the speed error; point-wise estimation can provide slightly better estimates of the wind speed at the expense of a higher direction error.

\section{Sample Results for Simulated NSCAT Data}

To further demonstrate the feasibility of model-based wind field estimation, we use the simulated mesoscale wind fields described in Part I [29], and Monte Carlo realizations of simulated measurements of $\sigma^{\circ}$ obtained by 


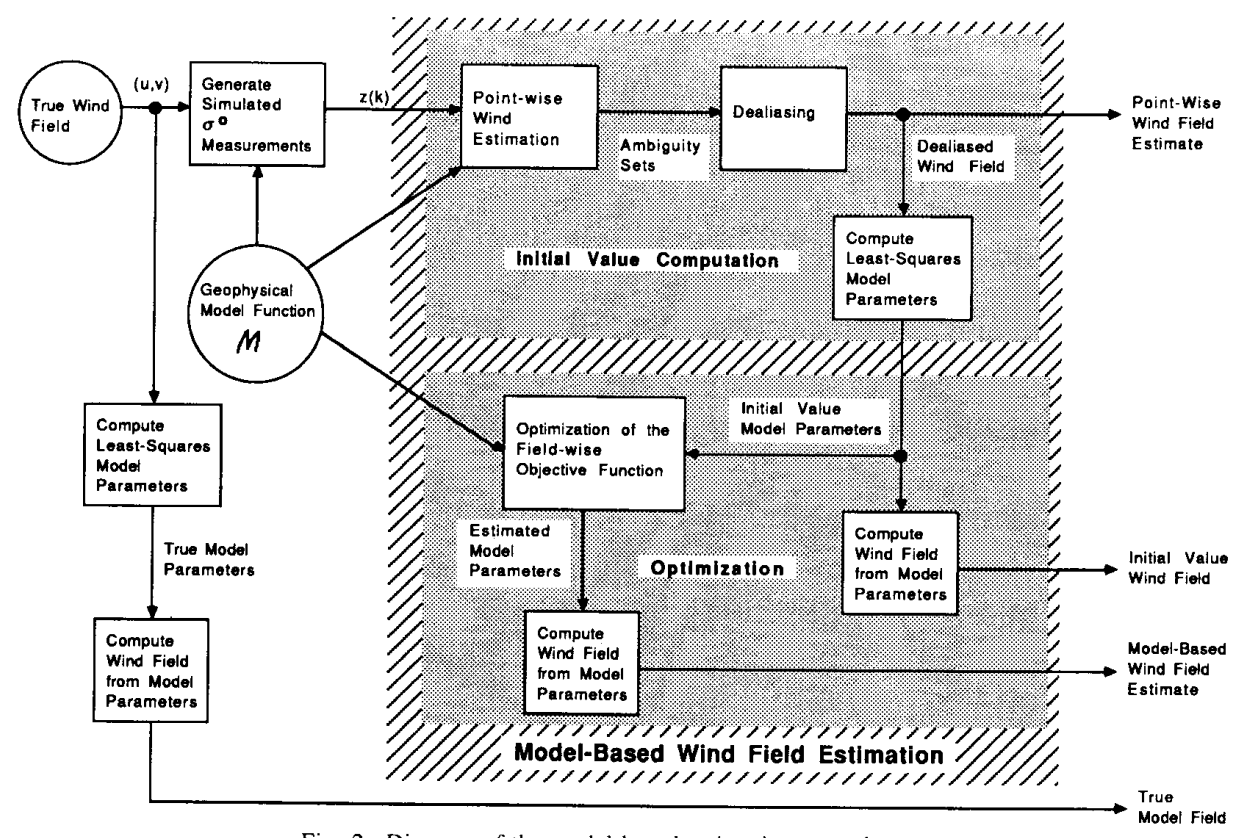

Fig. 2. Diagram of the model-based estimation procedure.

NSCAT [25]. A flowchart of the system simulation is shown in Fig. 2. We start with the "true", wind field (described in Part I [29]). Simulated $\sigma^{\circ}$ measurements are created using a state-of-the-art simulation of the NSCAT instrument, including all the effects of spacecraft-attitude control errors, uncertainty in the parameters of the radar equation used to compute $\sigma^{\circ}$ from the power measurements made by the scatterometer, and the uncertainty in the correct relationship between $\sigma^{\circ}$ and winds (i.e., geophysical modeling error in $\mathfrak{T})$. The simulation is the same which is used in [25].

The simulated $\sigma^{\circ}$ measurements are used to compute the point-wise wind estimates (ambiguity sets). This is followed by point-wise dealiasing using the median-filterbased dealiasing algorithm to compute the initial values for the field-wise objective function optimization. For each region of the measurement swath, initial value model parameters are computed using a least-squares fit (equation (8)). Starting with this initial value, the optimized model parameter vector is computed using the IMSL routine IMING. The final wind field estimate is computed using (7).

For model-based wind estimation, the left and right swaths are segmented in the along-track direction into 12 $\times 12(600 \times 600 \mathrm{~km})$ regions (see Fig. 3$)$. These region segments are indicated with dotted lines in the following figures. No continuity considerations between regions have been used, although this can simplify the computation [15].

Fig. 4 shows a wind field over both the left and right scatterometer swaths with an along-track distance of 3000 $\mathrm{km}$. The resolution is $50 \mathrm{~km}$ (see the discussion above). This will be the "true"' wind field in the results that fol-

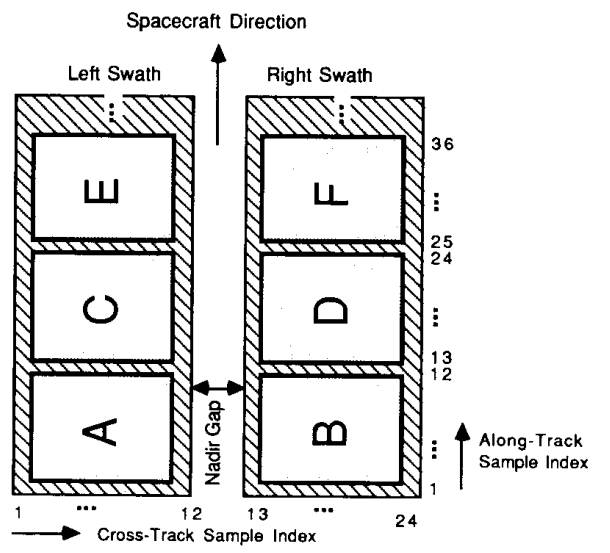

Fig. 3. Diagram of the region segmentation scheme for the $50-\mathrm{km}$ resolution example.

low. Fig. 5 shows the results of computing the leastsquare fit of the model parameters to the true field, and computing the resulting field on a region-by-region basis. This true model field represents the best the model-based estimation technique can achieve. For reference, Fig. 6 shows the closest alias to the true wind vector at each sample point, obtained from the ambiguity set using pointwise wind estimation. The results in Fig. 6 can only be obtained in a simulated example such as ours, because this field can only be obtained if the true field is known. This field would be the result of "perfect" dealiasing; actual dealiased results are worse due to dealiasing errors. The actual dealiased point-wise wind field estimate is shown in Fig. 7. Comparison of Figs. 6 and 7 reveals that in region $F$ the dealiased wind field has significant direc- 


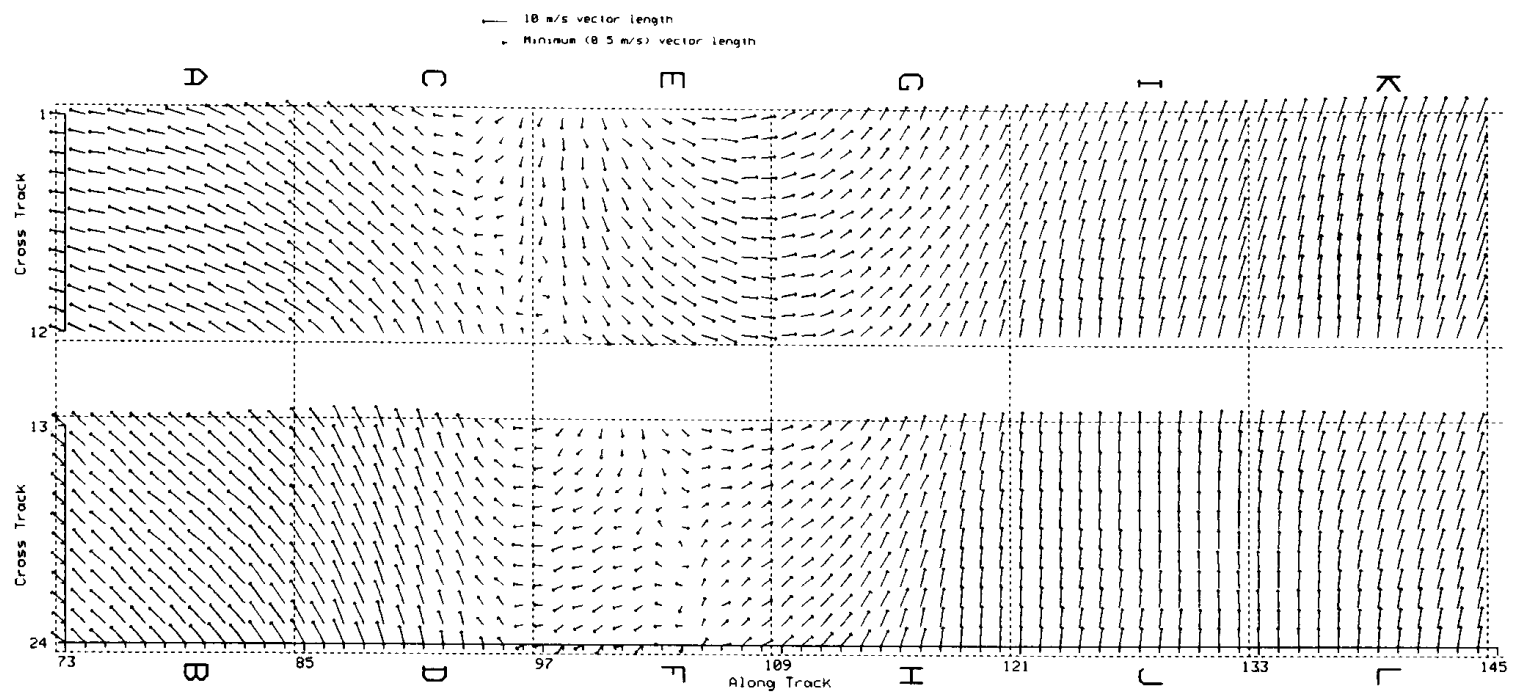

Fig. 4. True wind field over both NSCAT observation swaths. A special plotting convention has been used to enhance vector directions; wind speed is plotted on a linear scale between a maximum and minimum length chosen for clarity in indicating the direction of the minimum length vector. The maximum and minimum speed lengths are indicated in the figure.

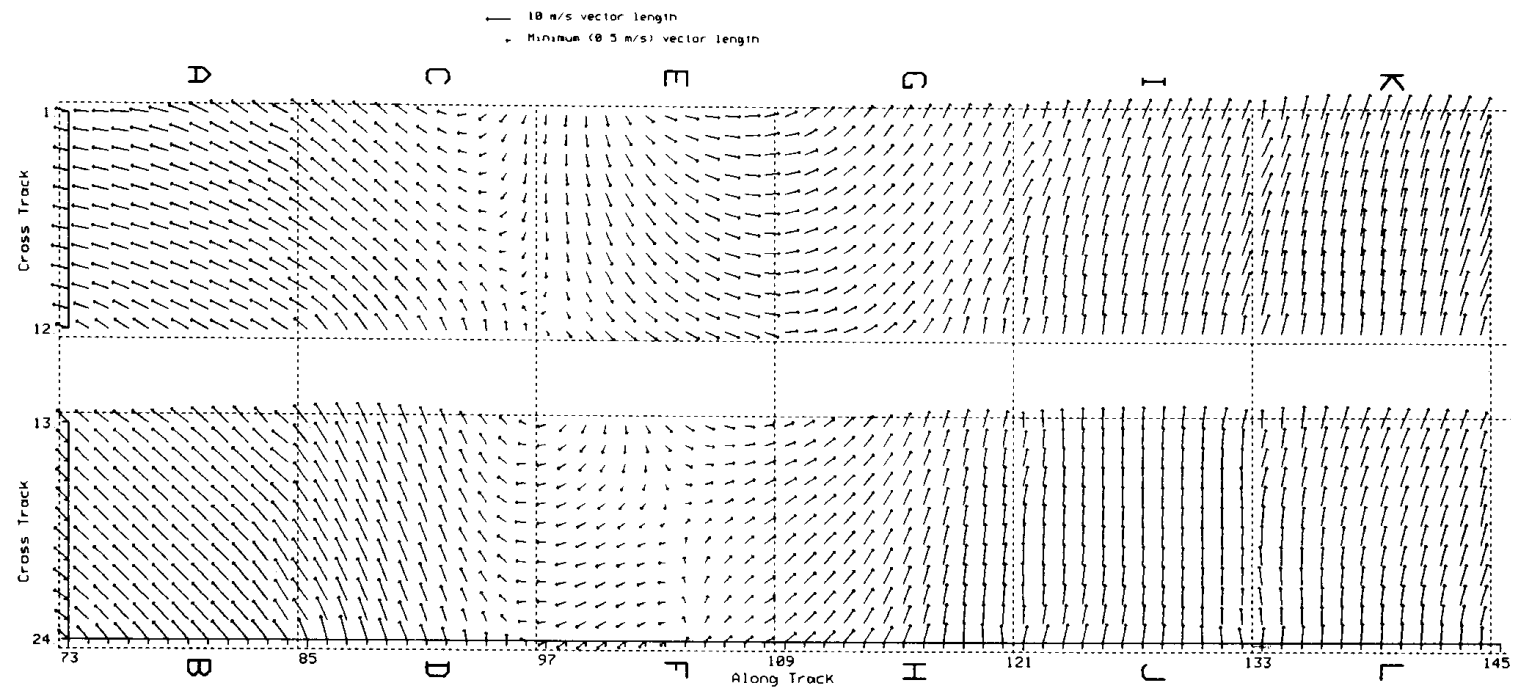

Fig. 5. True model field corresponding to Fig. 4. The plotting convention is the same as in Fig. 4.

tion errors. Note that in many regions the point-wise wind estimates (even the closest ambiguity field) appear to be very noisy. Fig. 8 shows the initial value wind field computed from Fig.7. The optimized wind field, which is the model-based estimate of the wind field, is shown in Fig. 9. The rms errors for selected regions are given in Tables II-VI. These regions were selected to span a variety of fields and results. They include regions of low wind speed and rapidly changing wind speed where dealiasing algorithms often perform poorly. Careful comparison of Figs. 4 and 7 will reveal that the wind field model effectively performs some "smoothing" of the wind field. The amount of smoothing and distortion in the region corners can be controlled by selection of the model type and order (see Part I [29]). Larger model orders minimize this smoothing effect and reduce the estimate error at the expense of additional computation during the optimization. This permits us to trade-off computational requirements and accuracy.

Again we note that the initial value field (Fig. 8) is a good estimate of the true wind field even without further optimization everywhere except in region $F$. In fact, the optimized field (shown in Fig. 9) does not look much different than the initial-value field except in region $F$, which 


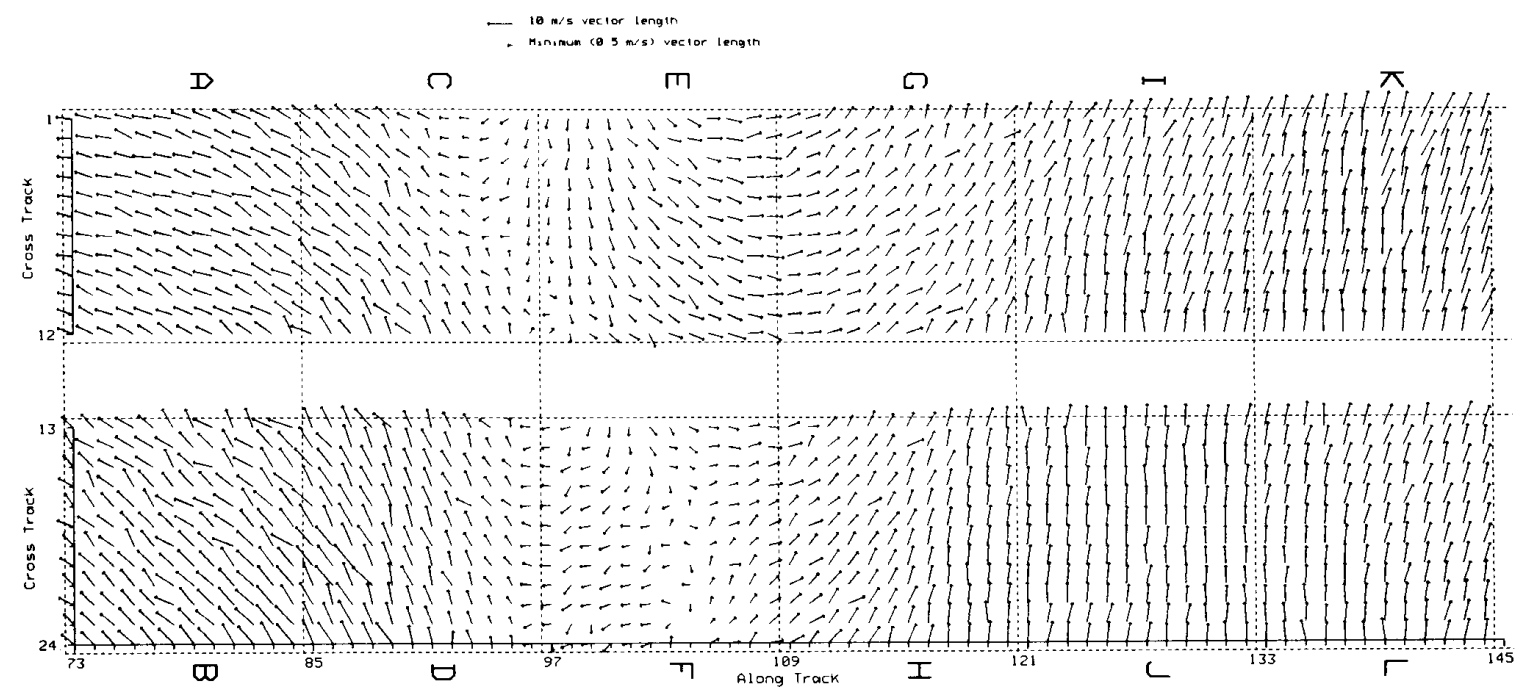

Fig. 6. Closest alias from the point-wise wind estimate ambiguity set to the true wind field. The point-wise ambiguity sets were determined using simulated NSCAT $\sigma^{\circ}$ measurements. Compare with Fig. 4 . The plotting convention is the same as in Fig. 4 .

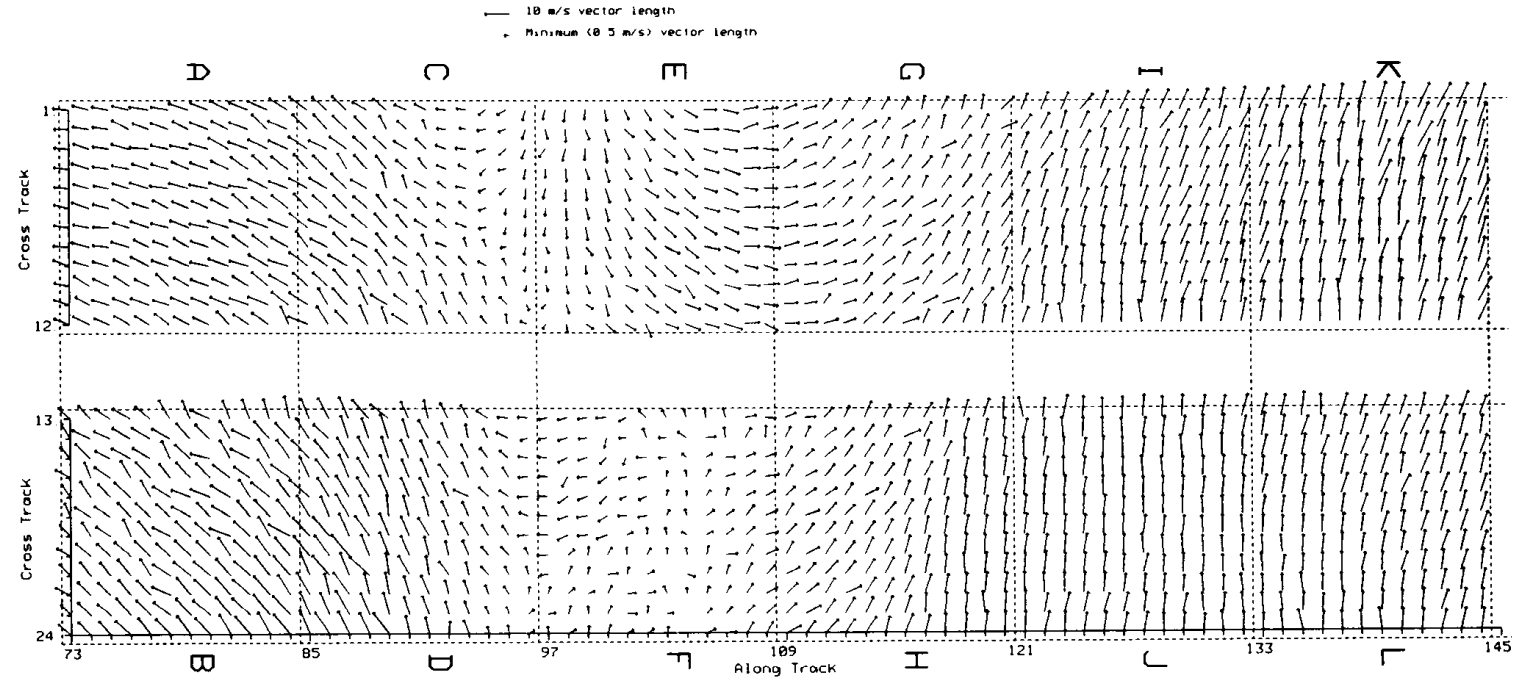

Fig. 7. Dealiased point-wise wind field estimate. Compare with Fig. 4. The plotting convention is the same as in Fig. 4.

will be considered below. This conclusion is strengthened by the rms error summary given on a region-by-region basis in Tables II-VI: With the exception of region $F$, just the process of initially fitting the wind field model to the point-wise wind field estimate dramatically improves the rms wind error. Optimization generally improves the rms error, although optimization of the objective function may increase the rms error slightly, since the objective function and rms error are different "cost" measures. Note that in virtually all regions the model-based estimates are better than the point-wise estimates even when perfect de- aliasing occurs. Table VII contrasts the total rms errors over all regions for the model-based (using a simplistic optimization scheme) and point-wise estimation results. The values given in the lower half of Table VII were computed with region $F$ excluded.

In region $F$, where large dealiasing errors were made, the initial model parameter vector was of very poor quality. While optimization improved the objective function value, the optimization algorithm stopped at a local minimum rather than at the global minimum. A more sophisticated optimization algorithm which finds the global min- 


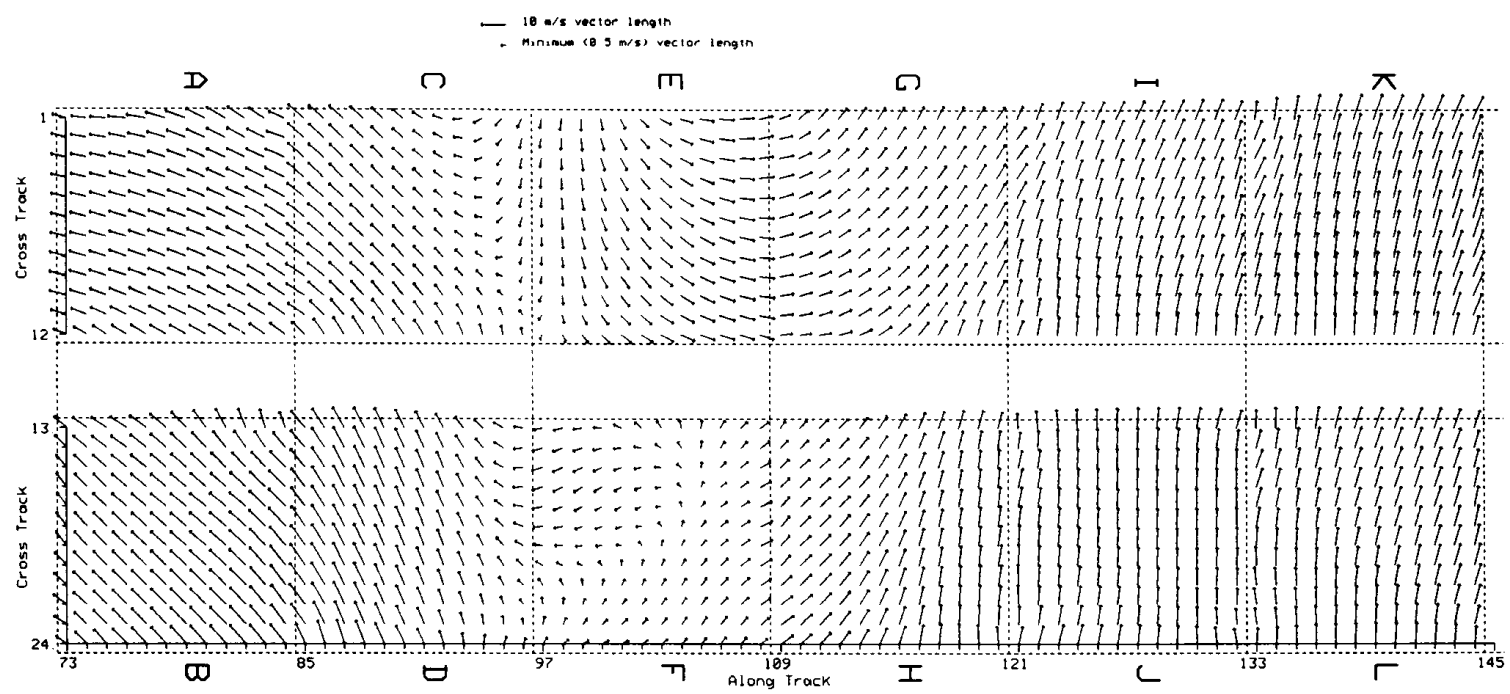

Fig. 8. The initial value wind field computed from Fig. 7. The plotting convention is the same as in Fig. 4.

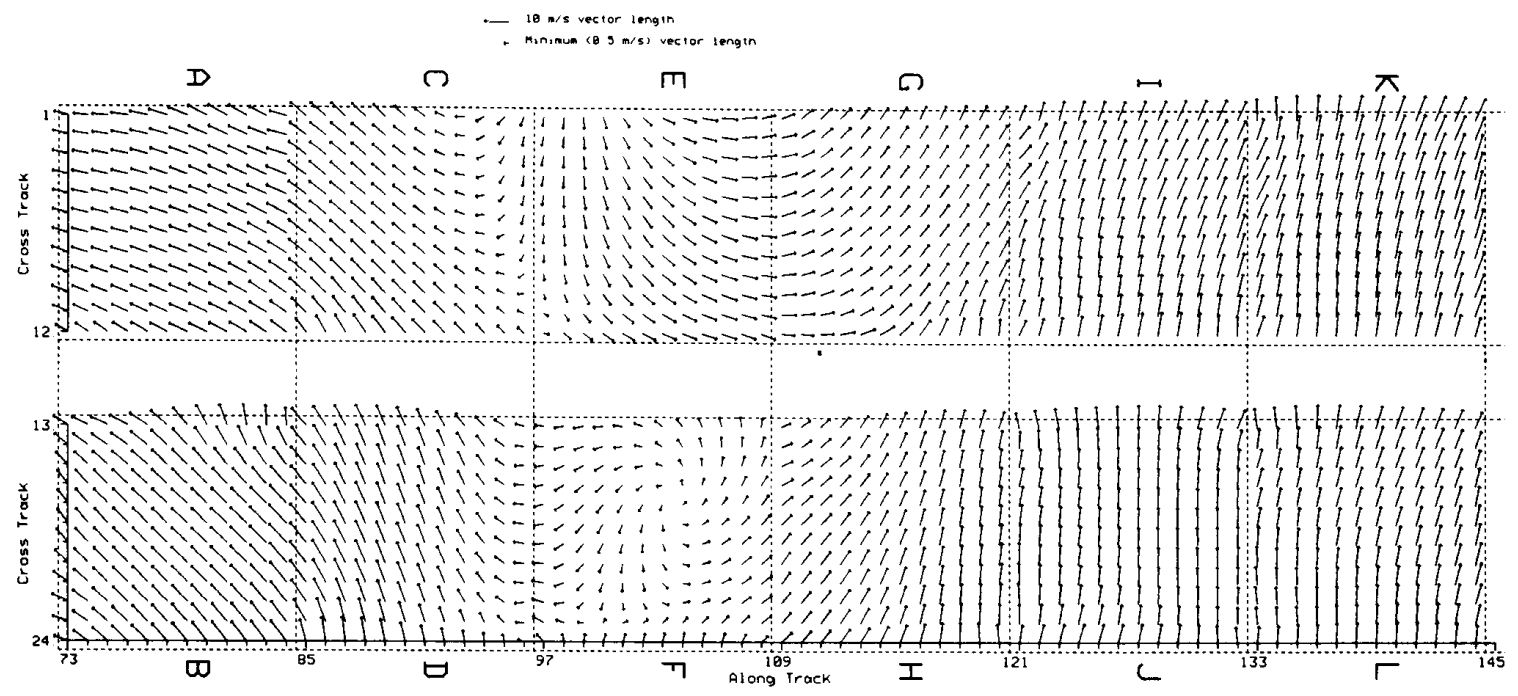

Fig. 9. The model-based wind field estimate computed from the optimized model parameters. Compare with Figs. 4 and 7 . The plotting convention is the same as in Fig. 4.

TABLE II

Region $A($ at 1,73$)$ Wind Estimation Performance

\begin{tabular}{|c|c|c|c|c|}
\hline Field & $\begin{array}{r}\text { Obj Func } \\
\text { Value }\end{array}$ & Vector & $\begin{array}{l}\text { RMS Err } \\
\text { Direction }\end{array}$ & Speed \\
\hline True Model & -5755.80 & 0.831 & 4.435 & 0.634 \\
\hline Point-wise Closest & - & 0.898 & 6.383 & 0.339 \\
\hline Point-wise Dealiased $^{\dagger}$ & - & 0.898 & 6.383 & 0.339 \\
\hline Initial Value & -5764.86 & 0.867 & 4.645 & 0.646 \\
\hline \multicolumn{5}{|l|}{ Optimized from: } \\
\hline Initial Value & -5879.98 & 0.968 & 5.577 & 0.651 \\
\hline True Model & -5951.15 & 0.967 & 5.576 & 0.651 \\
\hline
\end{tabular}

†Perfect dealiasing in this region.
TABLE III

Region $C$ (at 1,85 ) Wind Estimation Performance

\begin{tabular}{l|r|rrr}
\hline \hline Field & $\begin{array}{r}\text { Obj Func } \\
\text { Value }\end{array}$ & $\begin{array}{r}\text { RMS Err } \\
\text { Vector }\end{array}$ & Direction & Speed \\
\hline \hline True Model & -6319.14 & 0.783 & $\mathbf{1 3 . 1 7 8}$ & 0.521 \\
\hline Point-wise Closest & - & 1.105 & 13.577 & 0.344 \\
Point-wise Dealiased & - & 1.141 & 20.730 & 0.348 \\
\hline Initial Value & -6280.93 & 0.858 & 13.343 & 0.553 \\
\hline \multicolumn{5}{|c}{ Optimized from: } \\
\hline Initial Value & -6428.33 & 1.076 & 15.757 & 0.617 \\
True Model & -6428.37 & 1.081 & 15.690 & 0.614 \\
\hline
\end{tabular}


TABLE IV

Region $D$ (at 13,85$)$ Wind Estimation Performance

\begin{tabular}{|c|c|c|c|c|}
\hline Field & $\begin{array}{r}\text { Obj Func } \\
\text { Value }\end{array}$ & Vector & $\begin{array}{l}\text { RMS Err } \\
\text { Direction }\end{array}$ & Speed \\
\hline True Model & -5791.83 & 0.741 & 7.898 & 0.552 \\
\hline Point-wise Closest & & 1.260 & 10.327 & 0.513 \\
\hline Point-wise Dealiased & - & 1.361 & 24.953 & 0.528 \\
\hline Initial Value & -5722.53 & 0.913 & 18.905 & 0.584 \\
\hline \multicolumn{5}{|l|}{ Optimized from: } \\
\hline Initial Value & -5861.44 & 0.884 & 7.881 & 0.565 \\
\hline True Model & -5861.44 & 0.884 & 7.880 & 0.565 \\
\hline
\end{tabular}

TABLE V

Region $F($ at 13,97$)$ Wind Estimation Performance

\begin{tabular}{l|r|rrr}
\hline \hline Field & $\begin{array}{r}\text { Obj Func } \\
\text { Value }\end{array}$ & $\begin{array}{c}\text { RMS Err } \\
\text { Vector }\end{array}$ & Direction & Speed \\
\hline \hline Model Fit & -6740.41 & 0.691 & 26.257 & 0.444 \\
\hline Point-wise Closest & - & 0.872 & 23.012 & 0.344 \\
Point-wise Dealiased & - & 2.567 & 89.739 & 0.555 \\
\hline Initial Value & -5717.32 & 2.268 & 84.218 & 1.064 \\
\hline Optimized from: & \multicolumn{5}{|c}{} \\
\hline Initial Value & -6409.16 & 2.324 & 78.070 & 0.758 \\
True Model & -6841.41 & 0.811 & 27.058 & 0.446 \\
\hline
\end{tabular}

TABLE VI

Region $G($ at 1,109$)$ Wind Estimation Performance

\begin{tabular}{l|r|rrr}
\hline \hline Field & $\begin{array}{r}\text { Obj Func } \\
\text { Value }\end{array}$ & Vector & $\begin{array}{r}\text { RMS Err } \\
\text { Direction }\end{array}$ & Speed \\
\hline \hline True Model & -6158.89 & 0.574 & 4.356 & 0.417 \\
\hline Point-wise Closest & - & 1.196 & 12.006 & 0.293 \\
Point-wise Dealiased &.- & 1.198 & 12.039 & 0.292 \\
\hline Initial Value & -6128.49 & 0.711 & 6.056 & 0.440 \\
\hline Optimized from & \multicolumn{5}{|c}{} \\
\hline Initial Value & -6219.37 & 0.734 & 6.266 & 0.445 \\
True Model & -6219.37 & 0.732 & 6.236 & 0.446 \\
\hline
\end{tabular}

TABLE VII

Total Wind Field Estimate ERror

\begin{tabular}{|c|c|c|c|}
\hline \multirow[t]{2}{*}{ Field } & \multicolumn{3}{|c|}{ RMS Error } \\
\hline & Vector $(\mathrm{m} / \mathrm{s})$ & Direction (deg) & Speed $(\mathrm{m} / \mathrm{s})$ \\
\hline True Mode] & 0.669 & 10.033 & 0.485 \\
\hline Closest Ambiguity & 1.038 & 10.777 & 0.366 \\
\hline Point-wise Dealiased & 1.266 & 28.554 & 0.389 \\
\hline Initial Value & 0.994 & 26.079 & 0.578 \\
\hline Optimized from Initial Value & 1.098 & 24.243 & 0.539 \\
\hline Optimized from True Model & 0.874 & 10.946 & 0.508 \\
\hline Point-wise Dealiased $^{\dagger}$ & 1.072 & 12.544 & 0.370 \\
\hline Initial Value ${ }^{\dagger}$ & 0.781 & 9.856 & 0.511 \\
\hline Optimized from Initial Value $^{\dagger}$ & 0.907 & 9.331 & 0.514 \\
\hline Optimized from True Model ${ }^{\dagger}$ & 0.880 & 8.009 & 0.513 \\
\hline
\end{tabular}

†Region $F$ has been excluded.

imum results in a significantly more accurate estimate (discussed below). Even so, the model-based wind field estimate is better in an rms sense than the point-wise dealiased estimate. We note that the problem of stopping at a local minima can often be detected by comparing the wind speeds estimated using model-based estimation and the speeds estimated using point-wise estimation. When there is a significant difference, the model-based optimization has stopped at a local minimum.

Since the wind estimates shown above are based on a gradient-search optimization algorithm which uses a very simple technique for computing initial values, the optimization algorithm may get stuck in a local minimum; thus these results represent an upper bound on the performance of the model-based estimation technique. To demonstrate this, we used the true model parameters to initialize the gradient search. In all cases, the optimization, starting with the true model parameters, either locates the same (global) minimum as was previously found (starting with the initial-value field) or it locates a minimum with a lower value (i.e., the global minimum), which is closer to the true field than the initial-value field (see Tables IIVI). In these tables, compare the rms error and objective function values after optimization, starting with the initial value and true model value, respectively. Of particular interest is region $F$ (Table V). The optimized wind field obtained from starting the optimization with the true model parameters is shown in Fig. 10. These results indicate that improved initialization/optimization approaches (which find the global minimum) will yield even better estimates of the wind field. Our simple approach to initialization and optimization is, however, adequate for demonstrating the feasibility of model-based wind field estimation.

\section{Computational Considerations}

A disadvantage of the model-based estimation approach is that it requires significantly more computation than does the point-wise estimation approach, even for our simple initialization/approximation approach. If we require always locating the global minimum the computational requirements increase. We note that most of this time is consumed in optimizing the objective function. We have observed that if the initial-value wind fields have acceptable accuracy, we can save a significant amount of computation by not optimizing the objective function; i.e., by just using the initial-value wind field as our final result. We have observed that, in general, this accuracy is achieved if: (a) the rms of the estimated wind speed is larger than $4 \mathrm{~m} / \mathrm{s}$, and (b) the rms direction difference between the initial-value field and dealiased field is less than a threshold value (about $15^{\circ}$ ). These conditions can be checked before starting the optimization. If they are met, we can elect not to optimize and thereby trade off the accuracy of the estimate and computation time.

\section{Conclusion}

In this paper we have demonstrated the feasibility of model-based wind field estimation and have shown, by simulation, that even with simplistic initialization/optimization schemes, the model-based wind field estimates are more accurate than the point-wise wind estimates. Better results can be obtained by improved optimization schemes [15].

The methodology used in this research has application in other areas involving distributed parameter systems. In wind estimation we started with a well-defined measurement equation for a parameter $\left(\sigma^{\circ}\right)$, which is related to the quantity of interest (the wind) via a model function. Our approach to the estimation of the wind from measurements of $\sigma^{\circ}$ is to view the wind field as a distributed 


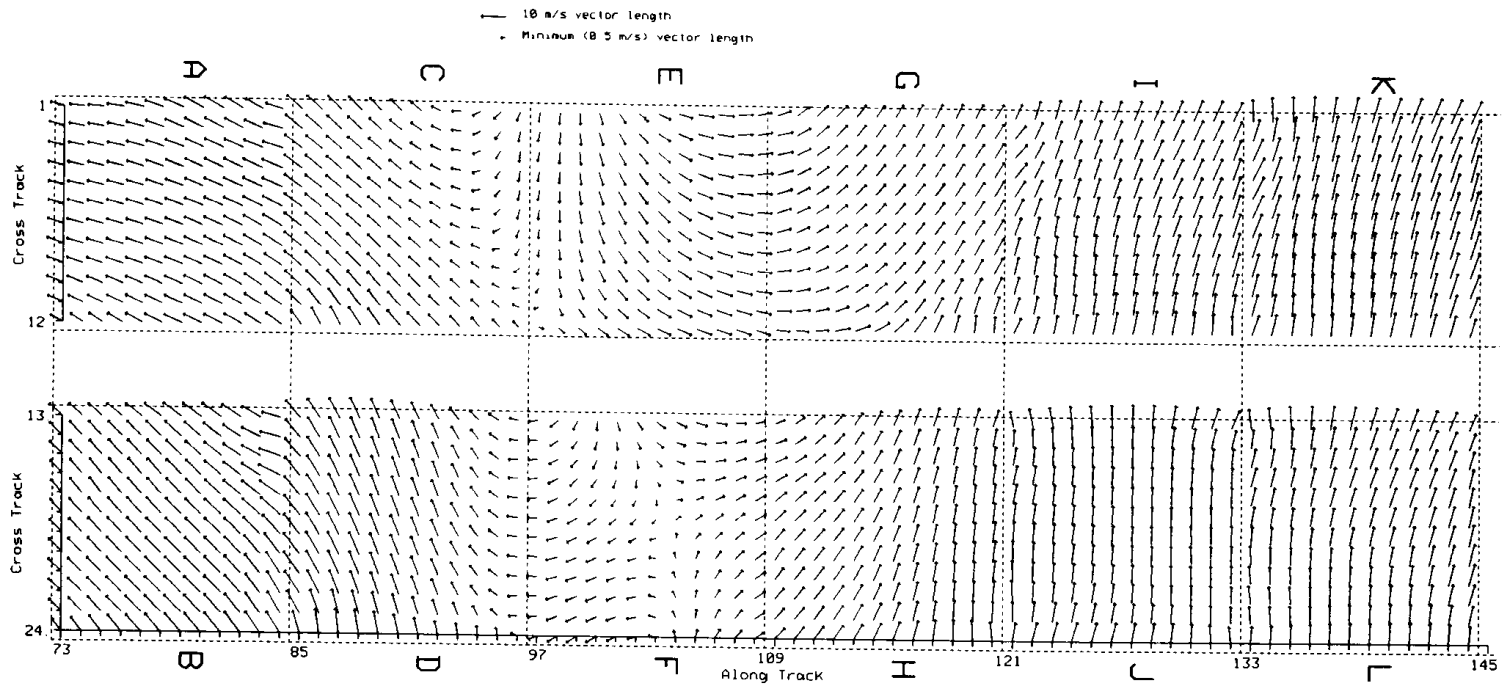

Fig. 10. The model-based wind field estimate computed from the optimized model parameters resulting from starting the optimization from the true model parameters. Note especially region $F$. Compare with Figs. 7 and 9 . The plotting convention is the same as in Fig. 4.

parameter system which can be approximately modeled by using partial differential equations. In effect, this system of equations provides constraints on the estimate of the quantity of interest, thus permitting more accurate estimates.

The partial differential equation system is solved by converting it to a finite-difference system, which is then arranged to express the quantity of interest in terms of a set of unknown parameters. This yields a simple model of the distributed parameter system. The quantity of interest is estimated indirectly by first estimating the unknown parameters directly from the measurements, then using the model to compute the quantity of interest from the estimated model parameters.

As the results of this research indicate, this methodology can be successfully used even when the relationship between the measured parameter and the desired quantity is nonunique, as is the case with the geophysical model function relating $\sigma^{\circ}$ and winds

This methodology has possible application in other remote sensing problems such as the estimation of atmospheric aerosols, surface topography, and wave height, as well as in such down-to-earth problems as the thermal control of steel in rolling mills.

\section{REFERENCES}

[1] D. Anderson et al., "A study of the feasibility of using sea and wind information from the ERS-1 satellite-Part 1: Wind scatterometer data," European Space Agency, Paris, France, ESA Contract Rep., June 1987.

[2] M. S. Bazaraa and C. M. Shetty, Nonlinear Programming-Theory and Algorithms. New York: Wiley, 1979.

[3] E. Bracalente, D. Boggs, W. Grantham, and J. Sweet, "The SASS scattering coefficient $\left(\sigma^{\circ}\right)$ algorithm, "IEEE J. Oceanic Eng., vol. OE-5, pp. 145-154, Apr. 1980.

[4] C.-Y. Chi, D. G. Long. and F. K. Li, "Radar backscatter measurement accuracies using digital Doppler processors in spaceborne scat- terometers," IEEE Trans. Geosci. Remote Sensing, vol. GE-24, pp. 426-437, May 1986

[5] C.-Y. Chi and F. K. Li, "A comparative study of several wind estimation algorithms for spaceborne scatterometers," IEEE Trans. Geosci. Remote Sensing, vol. 26, pp. 115-121,1988.

[6] R. E. Fischer, "Standard deviation of scatterometer measurements from space," IEEE Trans. Geosci. Electron., vol. GE-10, pp. 106113, Mar. 1972

[7] J. W. Johnson, L. A. Williams, Jr., E. M. Bracalente, F. B. Beck, and W. L. Grantham, "Seasat-A satellite scatterometer instrument evaluation," IEEE J. Oceanic Eng., vol. OE-5, pp. 138-144, Apr. 1980

[8] S. Kirkpatrick, C. D. Gelatt, Jr., and M. P. Vecchi, "Optimization by simulated annealing," Science, vol. 220, no. 4598, pp. 671-680, May 13, 1983.

[9] S. J. Leon, Linear Algebra with Applications. New York: Macmillan, 1980.

[10] F. K. Li, P. Callahan, M. Freilich, D, Long, and C. Winn, "NASA scatterometer for NROSS-a system for global oceanic wind measurement," in Proc. Int. Geosci Remote Sensing Symp. (Strasborg. France), 1984, pp. 777-780.

[11] L. Ljung, System Identification Theory for the User. Englewood Cliffs, NJ: Prentice-Hall, 1987.

[12] D. G. Long and J. M. Mendel, "Model-based estimation of wind fields over the ocean from wind scatterometer measurements." in Proc. Int. Geosci. Remote Sensing Symp. (Edinburgh, Scotland), 1988 , pp. 553-556.

[13] D. G. Long and J. M. Mendel, "Identifiability in wind estimation from scatterometer measurements." to be published.

[14] D. G. Long, C.-Y. Chi, and F. K. Li, "The design of an onboard digital Doppler processor for a spaceborne scatterometer," IEEE Trans. Geosci. Remote Sensing, vol. 26, pp. 869-878, Nov, 1988.

[15] D. G. Long, "Model-based estimation of wind fields over the ocean from wind scatterometer measurements," Ph.D. dissertation, Univ. of Southern California, Los Angeles, 1989

[16] D. G. Luenberger, Linear and Nonlinear Programming. Reading, MA: Addison-Wesley, 1984.

[17] B. D. Martin et al., "An overview of the NSCAT/N-ROSS program," in Proc. Workshop ERS-1 Wind and Wave Calibration (Schliersee, FRG), June 2-6, 1986, ESA SP-262, pp. 143-149.

[18] S. F. Masri, G. A. Bekey, and F. B. Safford, "A global optimization algorithm using adaptive random search," Appl. Math. Comput., vol. 7, pp. 353-375, 1980.

[19] J. M. Mendel, Discrete Techniques of Parameter Estimation. New York: Marcel Dekker, 1973. 
[20] J. M. Mendel, Lessons in Digital Estimation Theory. Englewood Cliffs, NJ: Prentice-Hall, 1987.

121] J. M. Mendel and K. S. Fu, Eds., Adaptive, Learning and Pattern Recognition Systems-Theory and Applications. New York: Academic, 1970

[22] R. K. Moore, R. G. Kennett, and F. K. Li, "Performance of a scanning pencil-beam spaceborne scatterometer for ocean wind measurements," in Proc. Int. Geosci. Remote Sensing Symp. (Edinburgh, Scotland), 1988, pp. 563-564.

[23] L. Schroeder et al., "Removal of ambiguous wind directions for a Ku-band wind scatterometer using measurements at three different azimuth angles," IEEE Trans. Geosci. Remote Sensing, vol. GE-23, pp. 91-100, Mar. 1985

[24] H. J. Schultz, "A median filter approach for correcting errors in a vector field," in Proc. Int. Geosci. Remote Sensing Symp. (Amhurst, MA), 1985, pp. 724-728.

[25] S. Shaffer, R. S. Dunbar, V. Hsiao, and D. G. Long, "A medianfilter-based ambiguity removal algorithm for NSCAT," IEEE Trans. Geosci. Remote Sensing, to be published.

[26] C. H. Slump and B. J. Hoenders, "The determination of the location of the global maximum of a function in the presence of several local extrema," IEEE Trans. Inform. Theory, vol. IT-31, pp. 490-497, July 1985 .

[27] F. T. Ulaby, R. K. Moore, and A. K. Fung, Microwave Remote Sensing-Active and Passive. Reading, MA: Addison-Wesley, 1981

[28] M. G. Wurtele et al., "Wind direction alias removal studies of SEASAT scattermeter-derived wind fields," J. Geophys. Res., vol 87, no. C5, pp. 3365-3377, Apr. 1982.

[29] D. G. Long and J. M. Mendel, "Model-based estimation of wind fields over the ocean from wind scatterometer measurements, part I: Development of the wind field model,"'IEEE Trans. Geosci. Remote Sensing, this issue, pp. 349-360.

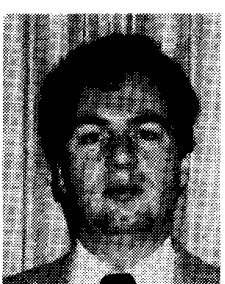

David G. Long ( $\left.S^{\prime} 80-M^{\prime} 82\right)$ received the B.S. and M.S. degrees in electrical engineering from Brigham Young University, Provo, UT, in 1982 and 1983, respectively, and the Ph.D. degree in electrical engineering from the University of Southern California, Los Angeles, in 1989.

He has worked for the Jet Propulsion Laboratory, Pasadena, CA. as a Radar Systems Engineer since 1983. He is currently the Project Engineer for the NASA Scatterometer (NSCAT) project and the Experiment Manager for the EOS SCANSCAT. NSCAT is a spaceborne scatterometer designed to measure oceanic winds from space. NSCAT is planned for flight in 1995 aboard the Japanese Advanced Earth Observing System (ADEos). SCANSCAT is a developmental scatterometer being studied for flight on NASA's Earth Observing System (EOS) in the late 1990's. He is responsible for the high level design, analysis, and technical management of these projects to insure that the projects meet all mission requirements. His responsibilities include overseeing instrument design and fabrication, algorithm, and coding for the ground processing system, mission operations, and data analysis. His technical responsibilities include system performance analysis, high-level design, development and maintenance of system requirements, and supervision of the Systems Engineering staff. $\mathrm{He}$ is also a Group Leader, supervising a staff of system engineers working on a number of JPL flight projects. His research interests include computer graphics, speech and signal processing, estimation theory, radar, and mesoscale atmospheric dynamics.

Dr. Long has received the NASA Award of Achievement several times.

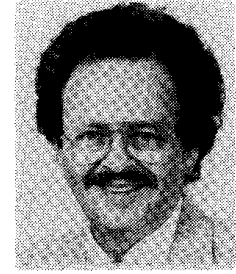

Jerry M. Mendel (S'59-M'61-SM'72-F'78) received the B.S. degree in mechanical engineering and the M.S. and Ph.D. degrees in electrical engineering from the Polytechnic Institute of Brook lyn, Brooklyn, NY, in 1959, 1960, and 1963, respectively.

His experience has included teaching courses in electrical engineering at the Polytechnic Institute of Brooklyn from 1960 to 1963 , and has also included various consulting positions. From July 1963 to January 1974 he was with the McDonnell-Douglas Astronautics Company. Currently, he is Professor and Chairman of Electrical Engineering-Systems at the University of Southern California, Los Angeles. He teaches courses in estimation theory and seismic data processing for oil exploration, and was Director of the USC GeoSignal Processing Program (1980-1983). He has published over 230 technical papers and is the author of the monographs: Maximum-Likelihood Deconvolution: A Journey into Model-Based Signal Processing (SpringerVerlag, 1990) and Optimal Seismic Deconvolution: An Estimation-Based Approach (Academic, 1983); the texts: Lessons in Digital Estimation The ory (Prentice-Hall, 1987) and Discrete Techniques of Parameter Estimation: The Equation Error Formulation (Dekker, 1973), and is co-editor (with K.S. Fu) of Adaptive, Learning and Pattern Recognition Systems (Academic, 1970). He is also author of the IEEE Individual Learning Program, Kalman Filtering, and Other Digital Estimation Techniques. $\mathrm{He}$ served as Editor of the IEEE Control Systems Society's IEEE TRANSAC TIONS ON AUTOMATIC CONTROL, for which he presently serves as an Associate Editor-at-Large, and is on the Editorial Board of the IEEE PROCEEDINGS.

Dr. Mendel is a member of the Society of Exploration Geophysicists, the European Association for Signal Processing, Tau Beta Pi, and Pi Tau Sigma, and is a registered Professional Control Systems Engineer in California. He was President of the IEEE Control Systems Society in 1986. He received the SEG 1976 Outstanding Presentation Award for a paper on the application of Kalman Filtering to deconvolution, the 1983 Best Transactions Paper Award for a paper on maximum-likelihood deconvolution in the IEEE Transactions on Geoscience and Remote Sensing, a Phi Kappa Phi book award for his research monograph on seismic deconvolution, a 1985 Burlington Northern Faculty Achievement Award, and a 1984 IEEE Centennial Medal. 